\title{
ON THE CONGRUENCES CONNECTED WITH CERTAIN MAGIC SQUARES*
}

\author{
BY \\ D. N. LEHMER
}

The familiar "uniform step" method of constructing magic squares with odd number of cells $\dagger$ depends on certain interesting congruences which seem not to have been studied. By means of them one is able to state in very simple form the necessary and sufficient conditions that a square constructed with a given "step" and a given "break-step" shall be "magic," "diabolic," or "symmetric" completely or in part.

A square containing the numbers $1,2,3, \cdots, n^{2}$ is said to be "magic" in the rows if the sum of the numbers in each row is the same. Since the sum of all the numbers in the square is $n^{2}\left(n^{2}+1\right) / 2$ the sum in each row must be $n\left(n^{2}+1\right) / 2$. Similarly for the columns. A "magic square" is magic in both rows and columns. If the numbers in the diagonal from the upper left hand corner to the lower right hand corner as well as the numbers in any two partial diagonals parallel to it which together make up $n$ numbers is the same the square is said to be magic in the positive diagonals. If it is also magic in the negative diagonals the square is called "diabolic." If further the sum of the two numbers in any two cells symmetrically placed with respect to the center cell is the same the square is said to be symmetric. As an illustration of a square which is magic, diabolic and symmetric one may take

$\begin{array}{rrrrrrr}14 & 23 & 39 & 6 & 15 & 31 & 47 \\ 18 & 34 & 43 & 10 & 26 & 42 & 2 \\ 22 & 38 & 5 & 21 & 30 & 46 & 13 \\ 33 & 49 & 9 & 25 & 41 & 1 & 17 \\ 37 & 4 & 20 & 29 & 45 & 12 & 28 \\ 48 & 8 & 24 & 40 & 7 & 16 & 32 \\ 3 & 19 & 35 & 44 & 11 & 27 & 36\end{array}$

The above square has been constructed by the "uniform step" method which may be described as follows:

* Presented to the Society, San Francisco Section, April 7, 1923, and June 12, 1926; received by the editors in January, 1929.

$\dagger$ See McClintock, On the most perfect form of magic squares, American Journal of Mathematics, vol. 19 (1897), p. 99. 
Denote the cell in the $p$ th column counting from the left and in the $q$ th row counting from the bottom by $(p, q)$. In the "uniform step" method of constructing magic squares one starts with the number 1 in the cell $(p, q)$. The number 2 is then placed in the cell $(p+\alpha, q+\beta)$, the number 3 in the cell $(p+2 \alpha, q+2 \beta)$, and so on, the numbers $p+k \alpha$ and $q+k \beta$ being reduced modulo $n$. It is clear that by this process the number $n+1$ will land in the cell $(p+n \alpha, q+n \beta)$ and this, modulo $n$, is the same as $(p, q)$ which is the cell already occupied by 1 . A "break-step" $(a, b)$ is then introduced which puts the number $n+1$ into the cell $(p+a, q+b)$. The original tep $(\alpha, \beta)$ is then resumed and the numbers $n+1, n+2, n+3, \cdots$, are entered in the cells $(p+a, q+b),(p+a+\alpha, q+b+\beta),(p+a+2 \alpha, q+b+2 \beta), \cdots$ On arriving at the number $2 n+1$ another resort to the break-step is necessary to avoid the already occupied cell $(p+a, q+b)$, and the numbers $2 n+1,2 n+2,2 n+3, \cdots$ are entered in the cells $(p+2 a, q+2 b),(p+2 a+\alpha, q+2 b+\beta),(p+2 a+2 \alpha$, $q+2 b+2 \beta), \cdots$. The square resulting from a continuation of this process is seen to depend entirely on the four numbers $\alpha, \beta, a, b$, and may or may not be magic, or if magic may or may not be diabolic or symmetric. We proceed to develope the necessary and sufficient relations between these four numbers to decide in advance the kind of square they indicate.

The result of the above process may be summed up in the following fundamental congruences for determining the cell $(A, B)$ in which a number $x$ is to be entered:

$A \equiv p+\alpha(x-1)+a\left[\frac{x-1}{n}\right], B \equiv q+\beta(x-1)+b\left[\frac{x-1}{n}\right](\bmod n)$,

where $(\alpha, \beta)$ is the "step" and $(a, b)$ is the "break-step," $(p, q)$ is the cell containing the number 1 and the symbol $[k]$ denotes the greatest integer in $k$. In the square given above for illustration $(\alpha, \beta)=(1,2),(a, b)=(3,5)$, $(p, q)=(6,4)$, and the position of any number $x$ is given by the congruences

$A \equiv 6+(x-1)+3\left[\frac{x-1}{7}\right], \quad B \equiv 4+2(x-1)+5\left[\frac{x-1}{7}\right](\bmod 7)$.

It is to be observed, in the first place, that the process may not fill all the cells of the square. In this connection we have the following theorem:

Given $\alpha, \beta, a, b$, all prime to $n$, the necessary and sufficient condition that the uniform step process shall fill the square is that the determinant ||$_{\beta b}^{\alpha a} \mid$ shall be prime to $n$.

For if two values $x_{1}$ and $x_{2}$ of $x$ should give the same cell $(A, B)$, then 


$$
\begin{array}{ll}
\alpha\left(x_{1}-x_{2}\right)+a\left\{\left[\frac{x_{1}-1}{n}\right]-\left[\frac{x_{2}-1}{n}\right]\right\} \equiv 0 & (\bmod n), \\
\beta\left(x_{1}-x_{2}\right)+b\left\{\left[\frac{x_{1}-1}{n}\right]-\left[\frac{x_{2}-1}{n}\right]\right\} \equiv 0 & (\bmod n),
\end{array}
$$

whence, since $b$ and $\beta$ are supposed prime to $n$,

$$
(\alpha b-\beta a)\left(x_{1}-x_{2}\right) \equiv 0
$$

and also

$$
(\alpha b-\beta a)\left\{\left[\frac{x_{1}-1}{n}\right]-\left[\frac{x_{2}-1}{n}\right]\right\} \equiv 0
$$

If now $(\alpha b-\beta a)$ is prime to $n$, congruence (2) shows that $x_{1}$ and $x_{2}$ lie between the same two multiples of $n$. For since the values of $[(n-1) / n]$ range between 0 and $n-1$ the congruence

$$
\left[\frac{x_{1}-1}{n}\right] \equiv\left[\frac{x_{2}-1}{n}\right]
$$

is also an equality. But if $x_{1}$ and $x_{2}$ lie between the same two multiples of $n$, congruence (1) is also an equality giving $x_{1}=x_{2}$ so that two numbers can not determine the same cell unless they are the same number. If however $n$ and $(\alpha b-\beta a)$ have a common divisor $\delta$ then we have

$$
\left[\frac{x_{1}-1}{n}\right] \equiv\left[\frac{x_{2}-1}{n}\right]
$$

$$
\left(\bmod \frac{n}{\delta}\right)
$$

and $x_{1} \equiv x_{2}(\bmod n / \delta)$. If then we put $x_{2}=x_{1}+(n / \delta) n$ this last congruence is satisfied. Put this value of $x_{2}$ in the other congruence and we have

$$
\left[\frac{x_{1}-1}{n}\right] \equiv\left[\frac{x_{1}-1}{n}+\frac{n}{\delta}\right]
$$

$$
\left(\bmod \frac{n}{\delta}\right)
$$

and since $n / \delta$ is an integer the congruence is clearly satisfied. Two values of $x$ which differ by $n^{2} / \delta$ will then fall in the same cell by this rule for filling the square and the square will therefore not be filled. In the theorems which follow this determinant is supposed to be prime to $n$ and the square is therefore filled.

THEOREM. If $\alpha$ and a are prime to $n$ the square is magic in the columns.

We show first the important fact that the numbers in any column form a complete system of incongruent numbers. For if two entries $x_{1}$ and $x_{2}$ occupy cells in the same column we must have 
$p+\alpha\left(x_{1}-1\right)+a\left[\frac{x_{1}-1}{n}\right] \equiv p+\alpha\left(x_{2}-1\right)+a\left[\frac{x_{2}-1}{n}\right]$

whence

$$
\alpha\left(x_{1}-x_{2}\right)+a\left\{\left[\frac{x_{1}-1}{n}\right]-\left[\frac{x_{2}-1}{n}\right]\right\} \equiv 0
$$

If now $x_{1} \equiv x_{2}$ then since $a$ is prime to $n$

$$
\left[\frac{x_{1}-1}{n}\right] \equiv\left[\frac{x_{2}-1}{n}\right]
$$

and, as before, this last congruence is also an equality and the two $x$ 's lie between the same two multiples of $n$. The congruence $x_{1} \equiv x_{2}$ is also an equality and $x_{1}=x_{2}$ contrary to hypothesis.

It is also clear that no two numbers in the same column can lie between the same two multiples of $n$, otherwise by the above congruence the two values of $x$ would be equal.

If now we write $x_{i}=k_{i} n+l_{i}$ where $0 \leqq k \leqq n-1$ and $0<l \leqq n$, then for the $x$ 's in any column the $k$ 's run through the values $0,1,2,3, \cdots, n-1$ and the $l$ 's through the values $1,2,3, \cdots, n$, so that

$$
\begin{aligned}
\sum x_{i}=n \sum k_{i}+\sum l_{i} & =\frac{n^{2}(n-1)}{2}+\frac{n(n-1)}{2} \\
& =\frac{n\left(n^{2}+1\right)}{2}
\end{aligned}
$$

and the square is thus seen to be magic in the columns. Similarly if $b$ and $\beta$ are prime to $n$ the square is magic in the rows. We have then the following theorem:

THEOREM. If $a, b, \alpha, \beta$ are all prime to $n$ the square is magic.

This condition is also necessary. To show this we prove first that if $a$ is prime to $n$ but $\alpha$ and $n$ have the greatest common divisor $\delta$, then the square is not magic in the columns. From the congruence obtained above for any two entries $x_{1}$ and $x_{2}$ in the same column

$$
\alpha\left(x_{1}-x_{2}\right)-a\left\{\left[\frac{x_{1}-1}{n}\right]-\left[\frac{x_{2}-1}{n}\right]\right\} \equiv 0 \quad(\bmod n)
$$

we can see that if $k$ is the smallest value of $[(x-1) / n]$ in the column then all the others in that column are given by $k+\rho_{i} \delta$ where $\rho_{i}=0,1,2, \cdots, n / \delta-1$. 
For if the congruence

$$
\alpha(x-1) \equiv A-p-a k
$$

has solutions, so will also the congruence

$$
\alpha(x-1) \equiv A-p-a\left(k+\rho_{i} \delta\right)
$$

Moreover for each value of $\rho_{i}$ there will be just $\delta$ solutions. Writing as above $x_{i}=k_{i} n+l_{i}$ it is clear that the values of $k_{i}$ fall into $\delta$ sets of $n / \delta$ numbers $k+\rho_{i} \delta$, so that

$$
\begin{aligned}
\sum k_{i}=\delta \sum k+\rho_{i} \delta & =\delta\left\{k \frac{n}{\delta}+\frac{\delta}{2}\left(\frac{n}{\delta}\right)\left(\frac{n}{\delta}-1\right)\right\} \\
& =k n+\frac{n}{2}(n-\delta) .
\end{aligned}
$$

As for the $l_{i}$, they form again a complete system of incongruent numbers. For if two were congruent then by the above reasoning the corresponding $x$ 's would be equal. The $l$ 's give again the series $1,2,3, \cdots, n$ in some order and $\sum l_{i}=n(n+1) / 2$. We have then for the sum of the $x$ 's in the column

$$
\begin{aligned}
\sum x_{i}=\sum k_{i} n+l_{i} & =k n^{2}+\frac{n^{2}}{2}(n-\delta)+\frac{n(n+1)}{2} \\
& =\frac{n}{2}\left(n^{2}+1\right)+n^{2}\left(k-\frac{\delta-1}{2}\right) .
\end{aligned}
$$

Now $k$, the smallest value of $[(x-1) / n]$ that will make $A-p-a[(x-1) / n]$ divisible by $\delta$, is seen to depend on $A$ and as $A$ takes the values $1,2,3, \cdots$, $n, k$ will run through the series of numbers $0,1,2, \cdots, \delta$ in some order, and two values of $A$ which are congruent modulo $\delta$ will give the same $k$. We have then the following theorem:

THEOREM. If $a$ is prime to $n$ but $\alpha$ and $n$ have the greatest common divisor $\delta$ then the square is not magic in the columns but the sum in any column differs from $(n / 2)\left(n^{2}+1\right)$ by some multiple of $n^{2}$ and column $A$ and column $A+\delta m$ have the same sum.

In the above formula if $\delta=1$ then $k=0$ and the sum of the $x$ 's in the column is $(n / 2)\left(n^{2}+1\right)$ as it should be.

A similar theorem holds, of course, for the rows when $b$ is prime to $n$ while $\beta$ and $n$ have the greatest common divisor $\delta$. As an illustrative example we take the square defined by $(\alpha, \beta)=(3,4),(a, b)=(2,3),(p, q)=(3,4), n=15$ : 


$\begin{array}{rrrrrrrrrrrrrrr}168 & 111 & 54 & 222 & 165 & 93 & 36 & 204 & 147 & 90 & 18 & 186 & 129 & 72 & 15 \\ 125 & 68 & 11 & 179 & 107 & 50 & 218 & 161 & 104 & 32 & 200 & 143 & 86 & 29 & 182 \\ 82 & 25 & 193 & 121 & 64 & 7 & 175 & 118 & 46 & 214 & 157 & 100 & 43 & 196 & 139 \\ 39 & 217 & 150 & 78 & 21 & 189 & 132 & 75 & 3 & 171 & 114 & 57 & 225 & 153 & 96 \\ 221 & 164 & 92 & 35 & 203 & 146 & 89 & 17 & 185 & 128 & 71 & 14 & 167 & 110 & 53 \\ 178 & 106 & 49 & 217 & 160 & 103 & 31 & 199 & 142 & 85 & 28 & 181 & 124 & 67 & 10 \\ 135 & 63 & 6 & 174 & 117 & 60 & 213 & 156 & 99 & 42 & 210 & 138 & 81 & 24 & 192 \\ 77 & 20 & 188 & 131 & 74 & 2 & 170 & 113 & 56 & 224 & 152 & 95 & 38 & 206 & 149 \\ 34 & 202 & 145 & 88 & 16 & 184 & 127 & 70 & 13 & 166 & 109 & 52 & 220 & 163 & 91 \\ 216 & 159 & 102 & 45 & 198 & 141 & 84 & 27 & 195 & 123 & 66 & 9 & 177 & 120 & 48 \\ 173 & 116 & 59 & 212 & 155 & 98 & 41 & 209 & 137 & 80 & 23 & 191 & 134 & 62 & 5 \\ 130 & 73 & 1 & 169 & 112 & 55 & 223 & 151 & 94 & 37 & 205 & 148 & 76 & 19 & 187 \\ 87 & 30 & 183 & 126 & 69 & 12 & 180 & 108 & 51 & 219 & 162 & 105 & 33 & 201 & 144 \\ 44 & 197 & 140 & 83 & 26 & 194 & 122 & 65 & 8 & 176 & 119 & 47 & 215 & 158 & 101 \\ 211 & 154 & 97 & 40 & 208 & 136 & 79 & 22 & 190 & 133 & 61 & 4 & 172 & 115 & 58\end{array}$

In accordance with the theorem the square is not magic in the columns, since the greatest common divisor of $\alpha$ and $n$ is 3. In accordance with a theorem to be proved it is also not magic in the rows because the greatest common divisor of $b$ and $n$ is also 3 . In the first column the smallest $k$ is 2 corresponding to the three entries $39,34,44$. The other $k$ 's are 5 , corresponding to the three entries $82,77,87$; and 8 corresponding to the entries $125,135,130$; and 11 corresponding to the entries $168,178,173$; and 14 corresponding to 221 , 216,211 . The sum in the first column is 1920 as given by the formula for $k=2, \delta=3$ and $n=15$. In the second column $k=1$ and the sum is 1695 by the formula. In the third column $k=0$ and the sum is 1470 . The sum is the same in the 1st, 4 th, 7 th, 10 th, and 13 th columns. Also in the $2 \mathrm{~d}, 5 \mathrm{th}, 8 \mathrm{th}$, 11 th, and 14th. Also in the 3d, 6th, 9th, 12th, and 15th.

We show now that if $\alpha$ is prime to $n$ but $a$ and $n$ have the greatest common divisor $\delta$, then the square is not magic in the columns. Starting with the same congruence which holds between two entries in the same column,

$$
\alpha\left(x_{1}-x_{2}\right)+a\left\{\left[\frac{x_{1}-1}{n}\right]-\left[\frac{x_{2}-1}{n}\right]\right\} \equiv 0 \quad(\bmod n),
$$

we see that $x_{1} \equiv x_{2}(\bmod \delta)$ and if $l$ is the smallest non-zero residue of the $x$ 's in the column, then all the $l$ 's in the column may be written $l+\rho_{i} \delta$ where $\rho_{i}=$ $0,1,2, \cdots, n / \delta-1$. We have then $\delta$ sets of numbers $l+\rho_{i} \delta$ and the sum of the $l$ 's in the column is

$$
\sum l_{i}=\delta \sum_{i=0}^{n / \delta-1}\left(l+\rho_{i} \delta\right)=n l+\frac{n(n-\delta)}{2} .
$$


As before the $k$ 's form a complete series of incongruent numbers running through the values $0,1,2, \cdots, n-1$. Their sum is therefore $n(n-1) / 2$. We thus get for the sum of the numbers in the column

$$
\begin{aligned}
\sum x_{i}=\sum k_{i} n+l_{i} & =\frac{n^{2}(n-1)}{2}+n l+\frac{n(n-\delta)}{2} \\
& =\frac{n}{2}\left(n^{2}+1\right)+n l-\frac{n(n-\delta)}{2} .
\end{aligned}
$$

As before it is clear that as the column $A$ is changed the values of $l$ run through the series of numbers $1,2,3, \cdots, \delta$ and any two columns $A$ and $A+\delta m$ will have the same $l$. It should be noted, however, that $l$ appears in the formula as multiplied by $n$ and not by $n^{2}$ so that the sums in the different columns will differ by multiples of $n$ and not as in the preceding case by $n^{2}$. We may then state the following theorem:

THEOREM. If $\alpha$ is prime to $n$ but $a$ and $n$ have the greatest common divisor $\delta$, then the square is not magic in the columns but the sum differs from $(n / 2)\left(n^{2}+1\right)$ by some multiple of $n$ and column $A$ and column $A+\delta m$ have the same sum.

A similar theorem holds of course for the rows when $a$ is prime to $n$ and $b$ and $n$ have the greatest common divisor $\delta$. In the illustrative example it is seen that the top row has the sum 1710 which comes from the formula by putting $n=15, \delta=3, l=3$. The same sum appears in the 4 th, 7 th, etc. rows from the top. The second row from the top adds to 1695 and the third to 1680 .

The preceding results may then be summed up in the following fundamental theorem:

THEOREM. The necessary and sufficient condition that the square obtained from the congruences

$$
\begin{aligned}
& A \equiv p+\alpha(x-1)+a\left[\frac{x-1}{n}\right] \\
& B \equiv q+\beta(x-1)+b\left[\frac{x-1}{n}\right]
\end{aligned}
$$

shall be magic is that the five numbers $\alpha, \beta, a, b$ and $(\alpha b-\beta a)$ shall be prime to $n$.

This theorem makes it clear why it is not possible to construct by the uniform step method a square with an even number of elements. In fact the five numbers indicated can not all be odd. It.should also be noted that the unit cell $(p, q)$ does not enter into the condition at all. We can therefore 
denote the above square by $\left(\begin{array}{c}\alpha a \\ \beta b\end{array}\right)$ with the understanding that the initial cell may be chosen arbitrarily.

Conditions for diabolism. If $A+B \equiv$ constant it is easily seen that the cells $(A, B)$ all lie in the same positive diagonal. Indeed, to keep the sum constant we must subtract from $B$ anything which we add to $A$. Similarly if $A-B \equiv$ constant the cells $(A, B)$ all lie in the same negative diagonal. Now we have

$$
\begin{aligned}
& A+B \equiv p+q+(\alpha+\beta)(x-1)+(a+b)\left[\frac{x-1}{n}\right], \\
& A-B \equiv p-q+(\alpha-\beta)(x-1)+(a-b)\left[\frac{x-1}{n}\right] \quad(\bmod n) .
\end{aligned}
$$

If therefore the numbers $\alpha+\beta, \alpha-\beta, a+b, a-b$, and $(\alpha+\beta)(a-b)-(\alpha-\beta)$ $\cdot(a+b)$ are prime to $n$ these congruences indicate a magic square whose columns are the positive diagonals and whose rows are the negative diagonals of the given square. We have then the following theorem:

THEOREM. The magic square $\left(\begin{array}{c}\alpha a \\ \beta b\end{array}\right)$ is diabolic if and only if the numbers $\alpha \pm \beta, a \pm b$ are prime to $n$.

(The condition that $(\alpha+\beta)(a-b)-(\alpha-\beta)(a+b)$ be prime to $n$ is fulfilled automatically if $\alpha b-\beta a$ is prime to $n$.) A curious corollary of this theorem is that a diabolic square cannot be constructed by the uniform step process when $n$ contains a factor 3 . For if $\alpha$ and $\beta$ are prime to $3, \alpha+\beta$ and $\alpha-\beta$ cannot both be. This does not mean, however, that a diabolic square cannot be obtained by other methods when $n$ is a multiple of 3 .

Conditions for symmetry. In a symmetrical square the sum of the two entries $x_{1}$ and $x_{2}$ in any two cells $(A, B)$ and $(1-A, 1-B)$ symmetrically situated with respect to the center is equal to $n^{2}+1$. This definition leads to the congruences

$$
\begin{aligned}
A & \equiv p+\alpha\left(x_{1}-1\right)+a\left[\frac{x_{1}-1}{n}\right], \\
B & \equiv q+\beta\left(x_{1}-1\right)+b\left[\frac{x_{1}-1}{n}\right], \\
1-A & \equiv p+\alpha\left(n^{2}+1-x_{1}-1\right)+a\left[\frac{n^{2}+1-x_{1}-1}{n}\right], \quad(\bmod n) . \\
1-B & \equiv q+\beta\left(n^{2}+1-x_{1}-1\right)+b\left[\frac{n^{2}+1-x_{1}-1}{n}\right] \quad
\end{aligned}
$$


The third and fourth simplify to

$$
\begin{aligned}
& 1-A \equiv p-\alpha x_{1}+a\left[n-\frac{x_{1}}{n}\right], \\
& 1-B \equiv q-\beta x_{1}+b\left[n-\frac{x_{1}}{n}\right]
\end{aligned}
$$

From these we get, with the first and second,

$$
\begin{aligned}
& 1 \equiv 2 p-\alpha+a\left\{\left[\frac{x_{1}-1}{n}\right]+\left[n-\frac{x_{1}}{n}\right]\right\}, \\
& 1 \equiv 2 q-\beta+b\left\{\left[\frac{x_{1}-1}{n}\right]+\left[n-\frac{x_{1}}{n}\right]\right\} \quad(\bmod n) .
\end{aligned}
$$

But we can show that

$$
\left[\frac{x_{1}-1}{n}\right]+\left[n-\frac{x_{1}}{n}\right]=n-1
$$

For suppose $\left(x_{1}-1\right) / n=k+\rho$ where $k$ is an integer and $0 \leqq \rho \leqq(n-1) / n$. Then the above expression is $k+[(n-k)-(\rho+1 / n)]$ and since $0<\rho+1 / n \leqq 1$, this is $k+n-k-1$ or $n-1$. The above congruences then become

$$
1 \equiv 2 p-\alpha-a, \quad 1 \equiv 2 q-\beta-b \quad(\bmod n),
$$

and we have the following theorem:

THEOREM. The magic square $\left(\begin{array}{c}\alpha a \\ \beta b\end{array}\right)$ is symmetric if and only if $2 p \equiv \alpha+a+1$, $2 q \equiv \beta+b+1(\bmod n)$.

The conditions for symmetry do not depend in any way upon the condition for a square to be magic, so that a square may be symmetric and not magic, as is exemplified in the above square of 15 . The central square pairs with itself and must be $\left(n^{2}+1\right) / 2$.

A more general type of diabolism arises by connecting the coördinates of the cells $(A, B)$ by a linear relation $\lambda A+\mu B \equiv$ constant $(\bmod n)$. Cells related in this way may be said to form a diagonal with a complementary diagonal given by $\lambda A-\mu B \equiv$ constant $(\bmod n)$. With these diagonals we have the

THEOREM. The square $\left(\begin{array}{c}\alpha a b \\ \beta b\end{array}\right)$ is diabolic with respect to the diagonals $\lambda A \pm \mu B$ if and only if the numbers $\lambda \alpha \pm \mu \beta, \lambda a \pm \mu b$ are prime to $n$.

Enumeration of uniform step squares. By means of the fundamental congruences defining uniform step squares it is easy to see that the tota number of such for a given prime $p$ is $p^{2}(p-1)^{3}(p-2)$. For we may choose 
the unit cell in $p^{2}$ different ways. Also the numbers $\alpha, \beta, a$ may be chosen at will from the $p-1$ numbers less than $p$. For each such choice there are $p-2$ choices of $b$ since the determinant $\alpha b-\beta a$ must be different from zero modulo $p$. The above result may be written $p^{2} \phi_{1}{ }^{3}(p)\left(\phi_{1}(p)-1\right)$ where $\phi_{1}$ is Euler's totient function.

For the case where the order of the square is a composite number $n$ the enumeration must be given more detailed consideration. In the congruence $\alpha b-\beta a \equiv k(\bmod n), \alpha, \beta$ and $a$ are given relatively prime to $n$; then as $k$ runs through a set of values prime to $n$ we are to find the number of $b$ 's which are also prime to $n$. This problem is equivalent to the following:

Let $\lambda$ be one of the numbers $\lambda_{n}$ which are less than a given number $n$ and prime to it. Find the number of numbers $\phi_{2}$ in the set $\lambda+\lambda_{n}$ which are prime to $n$.

Consider the complete set of numbers $1,2,3, \cdots, n$. We erase first of all the $n / p_{1}$ multiples of $p_{1}$, where $p_{1}$ is a prime divisor of $n$. When $\lambda$ is added to the set of numbers $1,2, \cdots, n$ we get again a complete set of incongruent numbers modulo $n$ from which again $n / p_{1}$ multiples of $p_{1}$ must be erased. For the prime $p_{1}$ we have therefore erased $2 n / p_{1}$ numbers from the list of numbers $1,2, \cdots, n$, leaving $n-2 n / p_{1}$. Similarly for the other prime factors of $n$. But any multiple of $p_{1} p_{2}$ has by this process been erased twice. We must then add $4 n /\left(p_{1} p_{2}\right)$, etc. By the well known principle of cross classification this gives at once the formula for $\phi_{2}$ :

$$
\phi_{2}=n-\sum \frac{2 n}{p_{1}}+\sum \frac{4 n}{p_{1} p_{2}}-\sum \frac{8 n}{p_{1} p_{2} p_{3}}+\cdots,
$$

which may be written in the form

or in the form

$$
\phi_{2}=n \Pi\left(1-\frac{2}{p_{i}}\right)
$$

$$
\phi_{2}=\Pi p_{i}^{\alpha_{i}-1}\left(p_{i}-2\right) \text {. }
$$

The formula gives zero for an even number which is as it should be, because in this case the numbers less than $n$ and prime to it are all odd and the sum of any two of them must be even and so not prime to $n$. As an illustration of the theorem take $n=45$. The set of numbers less than 45 and prime to it is $1,2,4,7,8,11,13,14,16,17,19,22,23,26,28,29,31,32,34,37,38,41,43,44$. Add to these any one of the list, say 11 , and erase all those that are not prime to 45 , and the nine numbers

$$
13,19,22,28,34,37,43,49,52
$$


are all that remain. The formula gives

$$
\phi_{2}(45)=3(3-2)(5-2)=9 .
$$

The above formula for $\phi_{2}$ is the same as that found by V. Schemmel (Journal für die reine und angewandte Mathematik, 1869, pp. 191-192) for the number of pairs of consecutive numbers each less than $n$ and both prime to $n$. It is seen by the above discussion that the numbers are not necessarily consecutive. Schemmel also gives the formula for the number of sets of $k$ consecutive integers all of which are prime to $n$, which is

$$
\phi_{k}=\Pi p_{i}^{\alpha_{i}-1}\left(p_{i}-k\right) \text {. }
$$

This formula is the enumerative function for the following much more general problem:

Given a set of $k$ linear forms $a_{1} x+b_{1}, a_{2} x+b_{2}, a_{3} x+b_{3}, \cdots, a_{k} x+b_{k}$, where the $a$ 's are all prime to $n$ and incongruent $\bmod n$. Let $x$ run through a complete set of incongruent numbers. Find the number of values of $x$ for which all the $k$ forms will give numbers prime to $n$.

To prove this formula we observe that as $x$ runs through a complete set of incongruent numbers so also does $a x+b$ and that there are $n / p_{1}$ values of $a x+b$ which are multiples of $p_{1}$ where $p_{1}$ is any prime factor of $n$. Each one of these values of $a x+b$ indicates a value of $x$ which is to be erased from the numbers $1,2,3, \cdots, n$. The $a$ 's being all supposed incongruent modulo $n$ we will thus have $k n / p_{1}$ numbers to erase from the set $1, \cdots, n$. The argument is thus the same as in the special case considered above. Many theorems regarding the ordinary totient function have their analogues for the more general function. Thus evidently if $m$ and $n$ are relatively prime we have $\phi_{k}(m) \phi_{k}(n)=\phi_{k}(m n)$.

The value of $\phi(1)$ being assumed equal to unity, the theorem The sum of totients of the divisors of $n$ is equal to $n$ carries over only when $n$ contains no square factor other than unity. But in this case we have the theorem

The sum of the $\phi_{k}$ functions of the divisors of $n$ is equal to $\phi_{k-1}$ function of $n$ when and only when $n$ has no square divisors other than unity.

We are now able to write down the number of magic squares obtainable by the uniform step method for any number $n=\Pi p_{i}{ }^{\alpha_{i}}$. The formula is

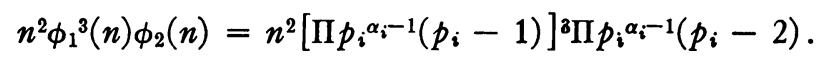

For symmetric squares we have no choice of the unit square. The formula for symmetric squares is therefore this result divided by $n^{2}$, or $\phi_{1}{ }^{3}(n) \phi_{2}(n)$. 
For diabolic squares we may choose $\alpha$ and $a$ at will from the $\phi_{1}(n)$ numbers less than $n$ and prime to it. Then $\beta$ must be chosen so that $\beta, \alpha+\beta$ and $\alpha-\beta$ shall be prime to $n$. This gives $\phi_{3}(n)$ choices for $\beta$. Finally $b$ must be chosen so that the four numbers $b, a+b, a-b, \alpha b-\beta a$ shall be prime to $n$. This number is $\phi_{4}(n)$. The formula, therefore, for the total number of diabolic squares obtainable by the uniform step method is

$$
\phi_{1}{ }^{2}(n) \phi_{3}(n) \phi_{4}(n)=\left[\Pi p_{i}^{\alpha_{i}-1}\left(p_{i}-1\right)\right]^{2} \Pi p_{i}^{\alpha_{i}-1}\left(p_{i}-3\right) \Pi p_{i}^{\alpha_{i}-1}\left(p_{i}-4\right) .
$$

This last formula points out the fact which we have noted before, that no diabolic square is obtainable by the uniform step method when $n$ is a multiple of 3. The following table gives the values of these functions for odd values of $n$ up to $n=35$.

$\begin{array}{rrrr}n & \text { Magic squares } & \text { Symmetric squares } & \text { Diabolic squares } \\ 3 & 72 & 8 & 0 \\ 5 & 4800 & 192 & 32 \\ 7 & 52920 & 1080 & 432 \\ 9 & 52488 & 648 & 0 \\ 11 & 1089000 & 9000 & 5600 \\ 13 & 3212352 & 19008 & 12960 \\ 15 & 345600 & 1536 & 0 \\ 17 & 17756160 & 61440 & 46592 \\ 19 & 35790984 & 99144 & 77760 \\ 21 & 3810240 & 8640 & 0 \\ 23 & 118288632 & 223608 & 183920 \\ 25 & 75000000 & 12000 & 20000 \\ 27 & 38263752 & 52488 & 0 \\ 29 & 498464064 & 592704 & 509600 \\ 31 & 752463000 & 783000 & 680400 \\ 33 & 78408000 & 72000 & 0 \\ 35 & 254016000 & 207360 & 13824\end{array}$

Transformations of magic squares. Certain transformations on the numbers 1 to $n^{2}$ have been devised which serve to throw a magic square into another magic square (see McClintock, loc. cit.). The question has not been studied as to whether the resulting square is a uniform step square or not. We proceed to answer this question by finding the connection between these transformations and the constants $\alpha, \beta, a, b$, which determine the square.

The numbers 1 to $n^{2}$ fall into $n$ sets as follows: 


$$
\begin{array}{ll}
K_{0} & (1,2,3, \cdots, n), \\
K_{1} & (n+1, n+2, \cdots, 2 n), \\
K_{2} & (2 n+1,2 n+2, \cdots, 3 n), \\
\cdot & \cdot \quad \cdot \quad \cdot \cdot \cdot \cdot \cdot \cdot \cdot . \\
K_{n-1} & \left(n(n-1)+1, n(n-1)+2, \cdots, n^{2}\right) .
\end{array}
$$

Let now $s$ be any substitution in the numbers 1 to $n$ such as

$$
s=\left(\begin{array}{ccc}
1, & 2, & 3, \cdots, n \\
x_{1}, & x_{2}, & x_{3}, \cdots, x_{n}
\end{array}\right)
$$

Perform now the same substitution on the numbers in each of the sets $K$. We get thus a transformation of the numbers 1 to $n^{2}$ which we denote by $S$. This transformation $S$, as we can show, will throw a uniform step square into a magic square which may or may not be a uniform step square. We have seen, in fact, that in a uniform step square each row (or column) contains one and only one number out of each of the above sets $K$, also that the numbers in any row (or column) form a complete series of incongruent numbers mqdulo $n$. Further, these two facts are sufficient to ensure that the square be magic. Now it is clear that neither of these conditions is disturbed by the transformation $S$, for the sets are not broken up, and also two entries in the same line can not be congruent to each other, for if $k n+x_{1}$ and $k n+x_{2}$ were congruent modulo $n$ so would also be $x_{1}$ and $x_{2}$ which is not so. The new square is therefore magic.

Again, let $T$ be any substitution on the sets $K$. Then again, if we start with a uniform step square the transformed square is still magic but not necessarily a uniform step square. For as before each set will furnish one and only one number in any row or column and those in any row or column will still furnish a complete set of incongruent numbers modulo $n$.

There are manifestly $(n !)^{2}$ transformed magic squares obtainable by $S$ and $T$ transformations from any given uniform step square. No two squares obtained from the same square by two different substitutions can be identical, for two different substitutions $S$ must make a different rearrangement of the numbers 1 to $n$ and so will give different squares. So also for two different transformations $T$. Moreover a transformation $S$ can not produce the same square as a transformation $T$ (except, of course, the identity transformation); for a transformation $T$ can not rearrange the numbers in the separate sets.

We consider now the following question: When will an $S$ or a $T$ transformation throw a uniform step square into another uniform step square?

Let $s$ be a substitution on the numbers 1 to $n$ such as 


$$
s=\left(\begin{array}{lll}
x_{1}, & x_{2}, & x_{3}, \cdots, \\
y_{1}, & y_{2}, & y_{3}, \cdots, y_{n}
\end{array}\right)
$$

where the $x$ 's and $y$ 's are correlated by the congruence $y \equiv \lambda x+\rho(\bmod n)$ where $\lambda$ is prime to $n$. Two values of $y$ give $y_{1}-y_{2} \equiv \lambda\left(x_{1}-x_{2}\right)(\bmod n)$ so that the difference between consecutive $y$ 's is $\lambda$ times the difference between the corresponding $x$ 's. This is equivalent to saying that the coefficients which determine the step have both been multiplied by a constant. The "breakstep" has, however, not been changed.

A transformation $T$ similarly defined makes a corresponding change in the coefficients of the "break-step" leaving the "step" unaltered. It is not difficult to see also that, if the difference between the $y$ 's is to be constant for consecutive $x$ 's, a congruential relation of the sort indicated above must hold. We have then the

THEOREM. If the transformation $S$ or $T$ is based on the substitution

$$
s=\left(\begin{array}{lll}
x_{1}, & x_{2}, & x_{3}, \cdots, \\
y_{1}, & y_{2}, & y_{3}, \cdots, y_{n}
\end{array}\right)
$$

then the necessary and sufficient condition that the resulting square be a uniform step square is that the $x$ and $y$ be related by the congruence $y \equiv \lambda x+\rho(\bmod n)$ where $\lambda$ is prime to $n$.

The above result may be stated as follows:

Those uniform step squares which are obtainable from a given square $\left(\begin{array}{l}\alpha a \\ \beta\end{array}\right)$ by an $S$-transformation are of the form $\left(\begin{array}{c}k \alpha, a \\ k, b\end{array}\right)$, and those obtainable by a T-transformation are of the form $\left(\begin{array}{l}\alpha, k^{\prime} a \\ \beta, k^{\prime} b\end{array}\right)$.

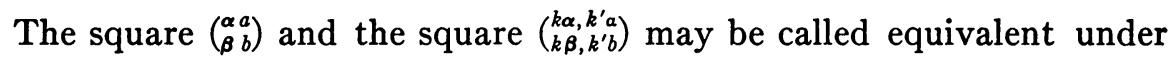
$H$ and $V$. Since the constants $k$ and $k^{\prime}$ may take $\phi_{1}(n)$ values, the number of squares equivalent to a given square form a set of $\phi_{1}{ }^{2}(n)$ squares. The total number of sets of non-equivalent squares is therefore $n^{2} \phi_{1}(n) \phi_{2}(n)$ or, if we consider symmetric squares only, $\phi_{1}(n) \phi_{2}(n)$. Thus, for example, the eight symmetric squares of three are in two non-equivalent sets:

$\begin{array}{llllllllllll}2 & 7 & 6 & 2 & 9 & 4 & 8 & 1 & 6 & 8 & 3 & 4 \\ 9 & 5 & 1 & 7 & 5 & 3 & 3 & 5 & 7 & 1 & 5 & 9 \\ 4 & 3 & 8 & 6 & 1 & 8 & 4 & 9 & 2 & 6 & 7 & 2 \\ 4 & 3 & 8 & 6 & 1 & 8 & 4 & 8 & 3 & 6 & 7 & 2 \\ 9 & 5 & 1 & 7 & 5 & 3 & 1 & 5 & 9 & 1 & 5 & 9 \\ 2 & 7 & 6 & 2 & 9 & 4 & 7 & 2 & 6 & 8 & 3 & 4\end{array}$.


From the fact that $k$ and $k^{\prime}$ are prime to $n$ it follows that if one of two equivalent squares is diabolic the other must be also. From the enumerative formula therefore the diabolic squares fall into $\phi_{3}(n) \phi_{4}(n)$ sets of non-equivalent squares. Thus the 32 diabolic squares of order 5 fall into 2 sets of 16 equivalent squares.

Any rearrangement of the rows or columns of a magic square will leave it magic since the sum in any row or column is thus left unaltered. A uniform step square, however, is not necessarily transformed in this way into a uniform step square. It appears easily that if the horizontal rows are transformed among themselves so that a row $x$ goes into a row $y$ where $x$ and $y$ are connected by the congruence relation $y \equiv \lambda x+\rho(\bmod n)$ where $\lambda$ is prime to $n$, then this transformation $H$ will throw a uniform step square into another uniform step square. It is found also that by this transformation the constants $\alpha$ and $a$ of the old square are left unaltered while $\beta$ and $b$ are multiplied by $\lambda$. Similarly a transformation $V$ of the same type on the columns throws a uniform step square into another uniform step square leaving the $\beta$ and $b$ unaltered and multiplying $\alpha$ and $a$ by $\lambda$. It follows that

Those uniform step squares which are obtainable for a given square $\left(\begin{array}{l}\alpha a \\ \beta\end{array}\right)$ by an $H$-transformation are of the form $\left(\begin{array}{c}\alpha a \\ k \beta k b\end{array}\right)$, and those obtainable by $a$ V-transformation are of the form $\left(\begin{array}{cc}k \alpha a & k a \\ \beta & b\end{array}\right)$.

The square $\left(\begin{array}{ll}\alpha & a \\ \beta & b\end{array}\right)$ and the square $\left(\begin{array}{ll}k \alpha & k a \\ k^{\prime} \beta & k^{\prime} b\end{array}\right)$ may be called equivalent under the transformations $H$ and $V$. It appears that an $H$-transformation followed by a $V$-transformation can not produce a square equivalent under $S$ and $T$ transformations unless the parameter $\lambda_{1}$ of the $H$ transformation equals the parameter $\lambda_{2}$ of the $V$ transformation. The squares, therefore, that are equivalent under all four types of transformations are of the form $\left(\begin{array}{l}k \alpha k a \\ k \beta\end{array}\right)$ and fall into sets of $\phi_{1}(n)$.

A third type of transformation is of use in the study of "nuclear" squares.* In this transformation the principal diagonals of the given square are made the row and column through the center of the new square. Any element of the old square lies in a cell which is the intersection of two diagonals which meet the principal diagonals in two elements $x_{1}$ and $x_{2}$. The corresponding cell in the new square for that element is found at the intersection of the row and column through those same elements $x_{1}$ and $x_{2}$. We proceed to set up the relations between the four numbers for the old and for the new cell.

Using the notions of elementary analytic geometry and calling the cell $(1,1)$ the origin, then the equation of the principal positive diagonal is found

\footnotetext{
* See John Willis, Easy Methods of Constructing Magic Squares, published by Percy Lund, Humphries and Co., London.
} 
to be $y=-x+1$ and of the principal negative diagonal is $y=x$. Also the positive diagonal through the cell $\left(A_{1}, B_{1}\right)$ has for its congruence $y-B_{1} \equiv$ $-\left(x-A_{1}\right)$. This meets the principal negative diagonal in the cell both of whose coördinates are $\frac{1}{2}\left(A_{1}+B_{1}\right)$. The negative diagonal through $\left(A_{1}, B_{1}\right)$ meets the principal positive diagonal in the cell whose coördinates are $\frac{1}{2}\left(A_{1}-B_{1}+1\right), \frac{1}{2}\left(B_{1}-A_{1}+1\right)$. Since now the cell $\left(A_{1}^{\prime}, B_{1}^{\prime}\right)$, the transform of $\left(A_{1}, B_{1}\right)$, must project on the principal row and column in those cells in which the diagonals through $\left(A_{1}, B_{1}\right)$ meet the principal diagonals in the first square, we have the coördinates $\left(A_{1}^{\prime}, B_{1}^{\prime}\right)$ given by

$$
A_{1}^{\prime}=\frac{A_{1}+B_{1}}{2}, \quad B_{1}^{\prime}=\frac{B_{1}-A_{1}+1}{2} .
$$

Assuming, now, that the first square is symmetrical, we have

$$
\begin{aligned}
& A_{1} \equiv \frac{1}{2}(\alpha+a+1)+\alpha x+a\left[\frac{x-1}{n}\right], \\
& B_{1} \equiv \frac{1}{2}(\beta+b+1)+\beta x+b\left[\frac{x-1}{n}\right]
\end{aligned}
$$

therefore

$$
\begin{aligned}
& A_{\mathrm{i}}^{\prime} \equiv \frac{1}{4}(\alpha+\beta+a+b+2)+\frac{\alpha+\beta}{2} x+\frac{a+b}{2}\left[\frac{x-1}{n}\right], \\
& B_{1}^{\prime} \equiv \frac{1}{4}(\beta+b-\alpha-a+2)+\frac{\beta-\alpha}{2} x+\frac{b-a}{2}\left[\frac{x-1}{n}\right] \quad(\bmod n) .
\end{aligned}
$$

The coefficients of the transformed square are therefore,

$$
\left(\begin{array}{cc}
\frac{\alpha+\beta}{2}, & \frac{a+b}{2} \\
\frac{\beta-\alpha}{2}, & \frac{b-a}{2}
\end{array}\right)
$$

It is clear from these coefficients that this square is magic in the diagonals if the original is magic in the rows and columns, and magic in the rows and columns if the original is magic in the diagonals. Thus a diabolic square goes by this transformation into a diabolic square. Since the general effect is a sort of twist through an angle of forty-five degrees, we shall speak of this transformation as a rotation and denote it by $R$. We give as illustration the square of eleven $\left(\begin{array}{ll}1 & 2 \\ 2 & 5\end{array}\right)$ and the transformed square $\left(\begin{array}{ll}7 & 9 \\ 6 & 7\end{array}\right)$ : 


$\begin{array}{rrrrrrrrrrr}103 & 86 & 58 & 41 & 13 & 117 & 89 & 72 & 55 & 27 & 10 \\ 94 & 77 & 49 & 32 & 4 & 108 & 80 & 63 & 35 & 18 & 111 \\ 85 & 57 & 40 & 12 & 116 & 99 & 71 & 54 & 26 & 9 & 102 \\ 76 & 48 & 31 & 3 & 107 & 79 & 62 & 34 & 17 & 121 & 93 \\ 56 & 39 & 22 & 115 & 98 & 70 & 53 & 25 & 8 & 101 & 84 \\ 47 & 30 & 2 & 106 & 78 & 61 & 44 & 16 & 120 & 92 & 75 \\ 38 & 21 & 114 & 97 & 69 & 52 & 24 & 7 & 100 & 83 & 66 \\ 29 & 1 & 105 & 88 & 60 & 43 & 15 & 119 & 91 & 74 & 46 \\ 20 & 113 & 96 & 68 & 51 & 23 & 6 & 110 & 82 & 65 & 37 \\ 11 & 104 & 87 & 59 & 42 & 14 & 118 & 90 & 73 & 45 & 28 \\ 112 & 95 & 67 & 50 & 33 & 5 & 109 & 81 & 64 & 36 & 19 \\ & & & & & & & & & & \\ 44 & 25 & 17 & 9 & 111 & 103 & 95 & 87 & 68 & 60 & 52 \\ 7 & 120 & 101 & 93 & 85 & 77 & 58 & 50 & 42 & 23 & 15 \\ 91 & 83 & 75 & 56 & 48 & 40 & 32 & 13 & 5 & 118 & 110 \\ 65 & 46 & 38 & 30 & 22 & 3 & 116 & 108 & 89 & 81 & 73 \\ 28 & 20 & 1 & 114 & 106 & 98 & 79 & 71 & 63 & 55 & 36 \\ 112 & 104 & 96 & 88 & 69 & 61 & 53 & 34 & 26 & 18 & 10 \\ 86 & 67 & 59 & 51 & 43 & 24 & 16 & 8 & 121 & 102 & 94 \\ 49 & 41 & 33 & 14 & 6 & 119 & 100 & 92 & 84 & 76 & 57 \\ 12 & 4 & 117 & 109 & 90 & 82 & 74 & 66 & 47 & 39 & 31 \\ 107 & 99 & 80 & 72 & 64 & 45 & 37 & 29 & 21 & 2 & 115 \\ 70 & 62 & 54 & 35 & 27 & 19 & 11 & 113 & 105 & 97 & 78\end{array}$

It should be noted that the principal positive diagonal might be taken as the row, or column, through the center of the new square, and that it might be taken to read either up or down for the column or right or left for the row. With these four ways of placing the principal positive diagonal will go also two ways of placing the principal negative diagonal in the new square, so that there should be eight different transformations of the type $R$ for a given square. The transformations for these eight cases are given in the following table:

Positive diagonal reading up and down, negative diagonal right and left:

Down, right,

Down, left,

$$
\left(\begin{array}{ll}
(\alpha+\beta) / 2 & (a+b) / 2 \\
(\beta-\alpha) / 2 & (b-a) / 2
\end{array}\right),
$$

$$
\left(\begin{array}{rr}
-(\alpha+\beta) / 2 & -(a+b) / 2 \\
(\beta-\alpha) / 2 & (b-a) / 2
\end{array}\right),
$$


Up, right,

Up, left,

$$
\left(\begin{array}{ll}
(\alpha+\beta) / 2 & (a+b) / 2 \\
(\alpha-\beta) / 2 & (a-b) / 2
\end{array}\right),
$$

$$
\left(\begin{array}{rr}
-(\alpha+\beta) / 2 & -(a+b) / 2 \\
(\alpha-\beta) / 2 & (a-b) / 2
\end{array}\right) .
$$

Positive diagonal right and left, negative diagonal up and down:

Right, down,

$$
\left(\begin{array}{rr}
(\alpha-\beta) / 2 & (a-b) / 2 \\
-(\alpha+\beta) / 2 & -(a+b) / 2
\end{array}\right)
$$

Left, down,

Right, up,

$$
\left(\begin{array}{rr}
(\beta-\alpha) / 2 & (b-a) / 2 \\
-(\alpha+\beta) / 2 & -(a+b) / 2
\end{array}\right)
$$

Left, up,

$$
\left(\begin{array}{ll}
(\alpha-\beta) / 2 & (a-b) / 2 \\
(\alpha+\beta) / 2 & (a+b) / 2
\end{array}\right),
$$

$$
\left(\begin{array}{ll}
(\beta-\alpha) / 2 & (b-a) / 2 \\
(\alpha+\beta) / 2 & (a+b) / 2
\end{array}\right) .
$$

It will be observed that these squares are paired in four ways such that the first and third as well as the second and fourth elements are interchanged. They are also paired in four ways such that the first and second change their signs, or the third and fourth change their signs. It is important to see how the numbers in the squares themselves are related.

Certain special transformations on symmetric squares. It is of interest to note the effect of various special transformations on the constants $\alpha, \beta, a$ and $b$ in symmetric squares. Thus it is easy to show that the two squares

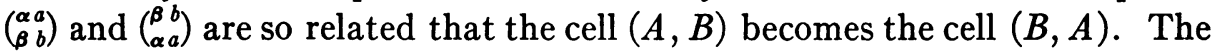
effect, therefore, on the square is to make a reflection in the main diagonal. This result appears at once from the fundamental congruences defining the two squares. The same method will also serve to show that the two

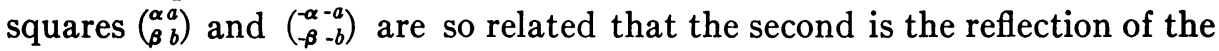
first in its center. For by writing out the congruences for the cell $(A, B)$ of the first square and for the corresponding cell (having the same $x$ ) in the second $\left(A^{\prime}, B^{\prime}\right)$ it appears easily that $A+A^{\prime} \equiv 1$ and $B+B^{\prime} \equiv 1(\bmod n)$. The effect is thus a rotation through two right angles about a line through the central cell perpendicular to the plane of the square. Simlarly $\left(\begin{array}{c}\alpha \\ -\beta\end{array}-b\right)$ is a reflection of $\left(\begin{array}{ll}\boldsymbol{\alpha} & a \\ \boldsymbol{\beta} & b\end{array}\right)$ in the row through the center. 
The result of a transformation may sometimes be conveniently expressed by means of a relation between the numbers in corresponding cells of the two squares. For this purpose a number $x$ is best represented in the form $x=k n+\rho$ where $k$ is one of the numbers $0,1,2,3, \cdots, n-1$ and $\rho$ is one of the numbers $1,2,3, \cdots, n$. It will be observed that $\rho$ is the smallest non-zero positive integer congruent to $x$ modulo $n$, and that $k$ is the number of the set (used in defining the transformation $S$ ) in which $x$ appears. If the number $x$ is divided by $n, k$ is the quotient and $\rho$ the remainder except for the case where $x$ is a multiple of $n$, in which case $k$ is the quotient less one and $\rho$ is equal to $n$.

It is possible to show for the two symmetric squares $\left(\begin{array}{c}\alpha a \\ \beta\end{array}\right)$ and $\left(\begin{array}{c}-\alpha a \\ -\beta\end{array}\right)$ that $x=k n+\rho$ occupies the same cell in the first square that $y=k n+n+1-\rho$ does in the second square. For calling the cell in which $x$ occurs $\left(A_{x}, B_{x}\right)$ and the cell of the second square in which $y$ occurs $\left(A_{y}^{\prime}, B_{y}^{\prime}\right)$, we have

$$
A_{x}=\frac{1}{2}(1+\alpha+a)+\alpha(k n+\rho-1)+a\left[\frac{k n+\rho-1}{n}\right],
$$

or, from the definitions of $k$ and $\rho$,

$$
A_{x}=\frac{1}{2}(1+\alpha+a)+\alpha(\rho-1)+a k ;
$$

similarly

$$
A_{y}^{\prime}=\frac{1}{2}(1-\alpha+a)-\alpha(-\rho)+a k .
$$

These two coördinates are manifestly the same. Similarly for the $B$ 's.

The two squares considered in the preceding paragraph are related by means of an $S$ transformation on the numbers in the sets as follows:

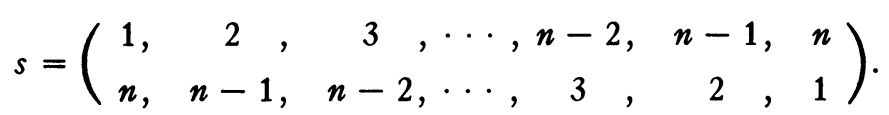

Any two numbers in corresponding cells in the two squares have the same $k$. There are $n$ numbers which occupy the same cell in both squares.

It is possible to show for the two symmetric squares $\left(\begin{array}{ll}\alpha & a \\ \beta & b\end{array}\right)$ and $\left(\begin{array}{l}a \alpha \\ b \beta\end{array}\right)$ that $x=k n+\rho$ occupies the same cell in the first that $y=(\rho-1) n+k+1$ does in the second. All that is necessary to prove this is to substitute the above value of $x$ in the congruences belonging to the first square and the above value of $y$ in the congruences belonging to the second.

These special theorems are of use in the study of "nuclear" squares to which we devote the next section. 
Nodal and nuclear magic squares. It is possible to show that there are magic squares which are not uniform step squares and which are not derivable from such squares by any of the transformations which have just been discussed. Such a square, for example, is the following:

$\begin{array}{rrrrrrrrrrr}36 & 17 & 119 & 89 & 32 & 51 & 70 & 2 & 104 & 85 & 66 \\ 16 & 118 & 99 & 69 & 1 & 31 & 50 & 103 & 84 & 65 & 35 \\ 117 & 98 & 68 & 49 & 102 & 11 & 30 & 83 & 64 & 34 & 15 \\ 97 & 67 & 48 & 29 & 82 & 101 & 10 & 63 & 44 & 14 & 116 \\ 26 & 7 & 109 & 79 & 42 & 60 & 81 & 43 & 13 & 115 & 96 \\ 46 & 27 & 8 & 110 & 100 & 61 & 22 & 12 & 114 & 95 & 76 \\ 77 & 47 & 28 & 9 & 41 & 62 & 80 & 113 & 94 & 75 & 45 \\ 6 & 108 & 78 & 59 & 40 & 21 & 112 & 93 & 74 & 55 & 25 \\ 107 & 88 & 58 & 39 & 20 & 111 & 92 & 73 & 54 & 24 & 5 \\ 87 & 57 & 38 & 19 & 121 & 91 & 72 & 53 & 23 & 4 & 106 \\ 56 & 37 & 18 & 120 & 90 & 71 & 52 & 33 & 3 & 105 & 86\end{array}$

It will be observed that the middle column contains the numbers 51 and 62 which are both congruent to 7 modulo 11 . It also contains the numbers 60 and 71 which are both congruent to 5 . But in a uniform step square the numbers in any row or column form a complete series of incongruent numbers, and any one of the transformations discussed above does not disturb this property of uniform step squares. It follows that the above square is not a uniform step square nor derivable from such a square by any $H, V, S$ or $T$ transformation.

It will be further observed that the above square is nuclear; that is, it has a central square (of three rows and columns in this case) in which the rows and columns add up to the same amount. We extend the definition of magic to include squares such as

$\begin{array}{rll}42 & 60 & 81 \\ 100 & 61 & 22 \\ 41 & 62 & 80\end{array}$

which are not made up of numbers beginning with unity, but which do have the same sum in the rows and columns. We say then that the above square has a magic nucleus of three. It is possible to find squares with a magic nucleus of five, or seven or of $m$ where $m$ is odd.*

Certain types of uniform step squares can be manipulated so as to produce nuclear squares. Thus the above nuclear square is obtainable from the symmetrical uniform step square

\footnotetext{
* For examples of such squares see John Willis, loc. cit.
} 


$$
A \equiv 6+x+\left[\frac{x-1}{11}\right], \quad B \equiv 9+x+7\left[\frac{x-1}{11}\right] \quad(\bmod 11)
$$

The central square for this is found to be

$\begin{array}{rrr}100 & 81 & 62 \\ 80 & 61 & 42 \\ 60 & 41 & 22\end{array}$

We perform now an $R$ transformation on this central square of 3 and get

$\begin{array}{rrr}42 & 60 & 81 \\ 100 & 61 & 22 \\ 41 & 62 & 80\end{array}$

The partial columns above the corners of this central square are then interchanged, and the partial rows to the left of the corners of this central square are also interchanged. The result is the nuclear square given above. The success of this method of getting nuclear squares is seen to depend on the fact that the central square of three is magic in its diagonals. In the same way we can obtain a nuclear square from any symmetrical uniform step square which has a central $m$-sided square which is magic in the diagonals. Since the square is symmetrical this common sum of all the diagonals must be $m\left(n^{2}+1\right) / 2$. Such a square we will call a nodal square. To obtain a nuclear square from such a nodal square make an $R$ transformation on the central square. Then the partial columns above and below the central square which are symmetrical in the nodal square must be so shifted that they lie in the same column. Thus, for example, the elements in the partial column above the upper left hand corner of the central square together with those in the partial column below the lower right hand corner of the central square add up to $(n-m)\left(n^{2}+1\right) / 2$. If these two partial columns are then shifted to lie in the same vertical column the sum of all the elements in that column will be $(n-m)\left(n^{2}+1\right) / 2+m\left(n^{2}+1\right) / 2$ or $n\left(n^{2}+1\right) / 2$, which is the right sum for a magic square. A similar shifting of the partial rows to the right and left of the central square will make the new square magic in all the rows. The problem of constructing nuclear squares by this method thus depends on the problem of finding symmetric uniform step squares which are nodal. It would seem possible to set up necessary and sufficient conditions on the constants of a uniform step square that it should be nodal, and perhaps from these conditions to obtain an enumeration of nodal squares. The author has not as yet been able to find such conditions, and the problem of enumeration of nodal squares seems to be of the same general type as the problem of finding the number of terms in a repeating decimal, or the number to which a given number belongs with respect to any modulus. He has suc- 
ceeded, however, in proving certain theorems which have enabled him to compute the number of such squares for a given side without much difficulty. Thus he has been able to prove that if $\left(\begin{array}{ll}\boldsymbol{\alpha} & a \\ \boldsymbol{\beta} & b\end{array}\right)$ is a nodal square we can write down at once the following list of nodal squares:

$$
\begin{aligned}
& \left(\begin{array}{ll}
\alpha & a \\
\beta & b
\end{array}\right)\left(\begin{array}{ll}
a & \alpha \\
b & \beta
\end{array}\right)\left(\begin{array}{ll}
\beta & b \\
\alpha & a
\end{array}\right)\left(\begin{array}{ll}
b & \beta \\
a & \alpha
\end{array}\right)\left(\begin{array}{rr}
-\alpha & -a \\
\beta & b
\end{array}\right)\left(\begin{array}{rr}
-a & -\alpha \\
b & \beta
\end{array}\right)\left(\begin{array}{rr}
-\beta & -b \\
\alpha & a
\end{array}\right)\left(\begin{array}{rr}
-b & -\beta \\
a & \alpha
\end{array}\right) \\
& \left(\begin{array}{ll}
-\alpha & a \\
-\beta & b
\end{array}\right)\left(\begin{array}{ll}
-a & \alpha \\
-b & \beta
\end{array}\right)\left(\begin{array}{ll}
-\beta & b \\
-\alpha & a
\end{array}\right)\left(\begin{array}{ll}
-b & \beta \\
-a & \alpha
\end{array}\right)\left(\begin{array}{rr}
\alpha & -a \\
-\beta & b
\end{array}\right)\left(\begin{array}{rr}
a & -\alpha \\
-b & \beta
\end{array}\right)\left(\begin{array}{rr}
\beta & -b \\
-\alpha & a
\end{array}\right)\left(\begin{array}{rr}
b & -\beta \\
-a & \alpha
\end{array}\right) \\
& \left(\begin{array}{ll}
-\alpha & -a \\
-\beta & -b
\end{array}\right)\left(\begin{array}{ll}
-a & -\alpha \\
-b & -\beta
\end{array}\right)\left(\begin{array}{ll}
-\beta & -b \\
-\alpha & -a
\end{array}\right)\left(\begin{array}{ll}
-b & -\beta \\
-a & -\alpha
\end{array}\right)\left(\begin{array}{rr}
\alpha & a \\
-\beta & -b
\end{array}\right)\left(\begin{array}{rr}
a & \alpha \\
-b & -\beta
\end{array}\right)\left(\begin{array}{rr}
\beta & b \\
-\alpha & -a
\end{array}\right)\left(\begin{array}{rr}
b & \beta \\
-a & -\alpha
\end{array}\right) \\
& \left(\begin{array}{ll}
\alpha & -a \\
\beta & -b
\end{array}\right)\left(\begin{array}{ll}
a & -\alpha \\
b & -\beta
\end{array}\right)\left(\begin{array}{ll}
\beta & -b \\
\alpha & -a
\end{array}\right)\left(\begin{array}{ll}
b & -\beta \\
a & -\alpha
\end{array}\right)\left(\begin{array}{rr}
-\alpha & a \\
\beta & -b
\end{array}\right)\left(\begin{array}{rr}
-a & \alpha \\
b & -\beta
\end{array}\right)\left(\begin{array}{rr}
-\beta & b \\
\alpha & -a
\end{array}\right)\left(\begin{array}{rr}
-b & \beta \\
a & -\alpha
\end{array}\right) .
\end{aligned}
$$

For certain values of $n$, of course, some of these may not be distinct. He has also been able to show that in a nodal square with a node of three, the entries in the columns and rows of the node are in actual arithmetical progression. This seems, experimentally, to hold for nodes of five and so on, but a proof is lacking for this. This theorem makes the enumeration of squares with a node of three a fairly simple matter even though an enumeration formula does not present itself. The number of nodal squares with a node of three for the first few values of $n$ are as follows:

$\begin{array}{rl}n & \text { number of squares } \\ 5 & 8 \\ 7 & 120 \\ 9 & 128 \\ 11 & 1648 \\ 13 & 3272 \\ 15 & 512 \\ 17 & 11712 \\ 19 & 19520 \\ 21 & 3072 \\ 23 & 46072 \\ 25 & 36424 \\ 27 & 12800\end{array}$

Since there are eight ways of performing the transformation $R$ on the central node and four ways of adjusting the partial columns above and below and to the right and left of the node to turn the square into a nuclear square, the above numbers must be multiplied by 32 for the number of nuclear squares with a nucleus of three obtainable by the above method. These nuclear squares are also still transformable into magic squares by the 
transformations $H$ and $V$ on the rows and columns, in number $(n !)^{2}$. The transformations $S$ and $T$ are however not available for squares of this sort.

The study of nodal squares brings to light many curious and interesting theorems of the following type:

The square $\left(\begin{array}{l}1 \\ 1\end{array} \frac{1}{2}\right)$ is nodal for all values of $n$. The square $\left(\begin{array}{l}1 \\ 1 \\ 1\end{array}\right)$ is nodal for all values of $n$ prime to 6 . The square $\left(\begin{array}{l}1 \\ 15\end{array}\right)$ is nodal for $n=4 k+1$, but not for $n=4 k-1$. The square $\left(\begin{array}{l}1 \\ 1\end{array}\right)$ is nodal for numbers of the form $14 k-$ 1 and for no others. The square $\left(\begin{array}{c}4 \\ 3\end{array}\right)$ s $)$ is never nodal for any value of $n$, etc.

UNIVERSITY OF CALIFORNIA, Berkeley, Calif. 